\title{
Joule-heated Molten Regolith Electrolysis Reactor Concepts for Oxygen and Metals Production on the Moon and Mars
}

\author{
Laurent Sibille $^{1}$ and Jesus A. Dominguez ${ }^{2}$ \\ ESC-Team QNA, Kennedy Space Center, FL, 32899
}

\begin{abstract}
The technology of direct electrolysis of molten lunar regolith to produce oxygen and molten metal alloys has progressed greatly in the last few years. The development of longlasting inert anodes and cathode designs as well as techniques for the removal of molten products from the reactor has been demonstrated. The containment of chemically aggressive oxide and metal melts is very difficult at the operating temperatures ca. $1600{ }^{\circ} \mathrm{C}$. Containing the molten oxides in a regolith shell can solve this technical issue and can be achieved by designing a Joule-heated (sometimes called 'self-heating') reactor in which the electrolytic currents generate enough Joule heat to create a molten bath. Solutions obtained by multiphysics modeling allow the identification of the critical dimensions of concept reactors.
\end{abstract}

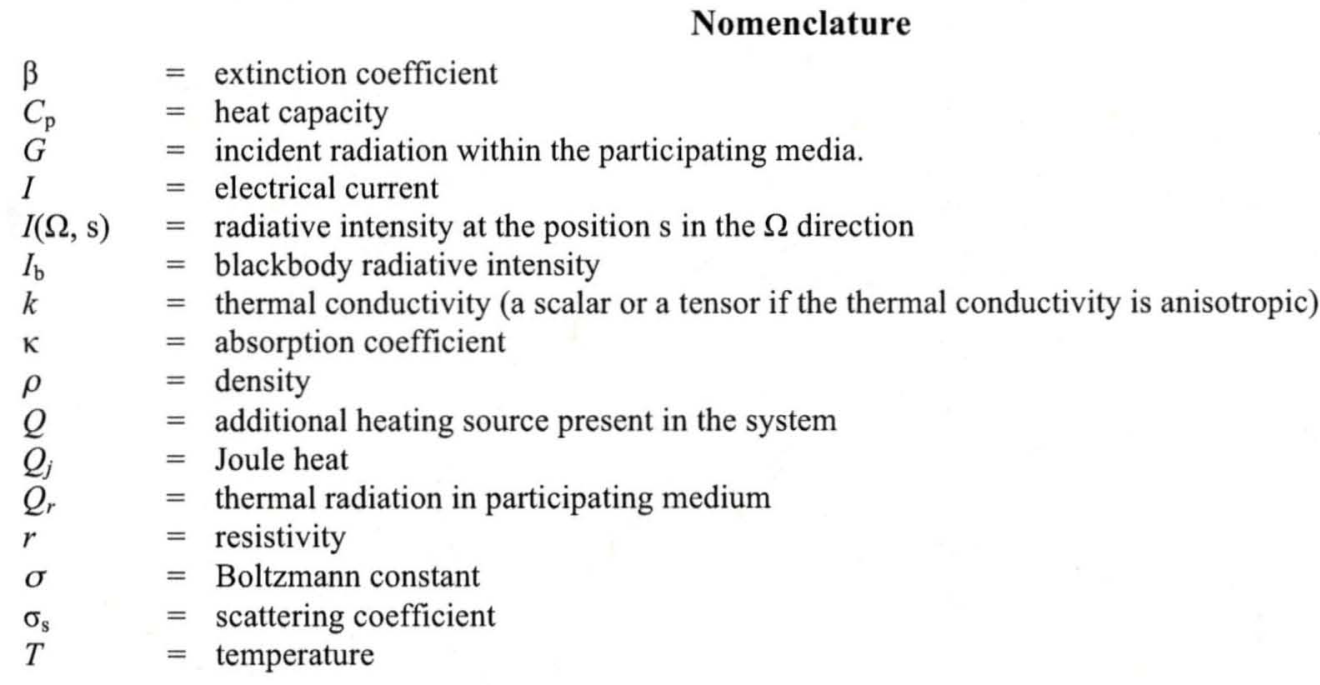

\section{Introduction}

THE maturation maturation of Molten Regolith Electrolysis (MRE) as a viable technology for oxygen and metals production on explored planets relies on the realization of the internal-heating mode for the reactor. In this configuration, the passage of current through the melt between the electrodes during electrolysis creates enough thermal energy by Joule effect to maintain the molten pool of oxides and metals. Analogous to the operations of the Hall-Héroult cell for aluminum production, this configuration offers many advantages: it makes use of the regolith itself as a crucible material thus protecting the vessel walls and simplifying the engineering of the reactor by eliminating the need for high-temperature materials for containment. The self-heating mode also reduces the energy needed for maintaining the regolith molten by eliminating the need for external heating.

The modeling of the self-heating mode is a valuable tool in the technology development cycle. It is designed to verify the feasibility of the containment of molten material and to identify dimensional parameters for the design

\footnotetext{
${ }^{1}$ Technology Development Scientist, Applied Science \& Technology, M/S ESC-24, AIAA Associate Fellow.

${ }^{2}$ Senior Engineer, Applied Science \& Technology, M/S ESC-24, AIAA Member. 
of a self-heating reactor. We selected the COMSOL Multiphysics ${ }^{\mathrm{TM}}$ modeling suite for the advantages in versatility and costs it offered. This work presents the development of the heat transfer calculations, the required properties of the regolith, and the validation of the model by reproducing the experimental results of an irradiated half-sphere of regolith by a concentrated solar beam. Preliminary predictions of self-heating and startup modes complete this report.

\section{Background}

The concept of high temperature electrolysis of oxides is used industrially in the production of Aluminum and Magnesium in which the natural oxides of these metals are selected in high purity form, dissolved in molten halide salts, and are electrolytically dissociated to electrowin molten metal as a product and oxygen as a by-product. In practice, the oxygen is evolved as carbon dioxide and monoxide through the use of carbon anodes. These technologies work remarkably well thanks to the tailored dissolution of these pure oxides in specific formulas of halide salts (cryolite in the case of aluminum production), although it is worth noting that the saturation limits of oxides in those tailor-made salts is around 4-5 wt.\%.

Electrowinning of metal and oxygen from multi-oxide mixtures and particularly from natural mineral mixtures using salts as dissolving media is very limited because of the inherent low dissolving power of these salt media. Since molten halide salts are formulated to solubilize one oxide typically, their solvating performance is quite inefficient in the presence of many oxides. The electrolysis of oxides in their own melt eliminates the obstacle of dissolution into a different electrolyte. It also allows a much more beneficial ratio of feed mass to reactor mass, an important parameter for space-bound hardware.

Specific techniques have been developed in collaboration with D.R. Sadoway's group at Massachusetts Institute of Technology (MIT) to perform measurements of the electrochemical behavior of high temperature molten oxides ${ }^{1}$. Gmitter et al. at MIT also showed that optical basicity, used in glassmaking is a concept that provides a measure of the total available oxygen-containing molecular anions as a function of the composition of the oxide melt ${ }^{2}$. The work shows the strong correlation between the calculated optical density of a melt of a given composition and the expected current density that the melt can support during electrolysis. The experimental results have confirmed these findings where current densities of the order of $0.5 \mathrm{~A} / \mathrm{cm}^{2}$ were recorded at $6 \mathrm{~V}$ for currents near 5 to $10 \mathrm{~A}$.

The current configuration and dimensions of the molten regolith electrolytic reactors forces their operation as hot-walls reactors. In fact, the oxide melt must be maintained in a superheated molten state through external heating provided by the resistive furnace in our experiments. Such conditions place particularly severe constraints on materials used to contain both the melt, the molten metal pooling at the bottom, and the evolving oxygen. Very few materials can perform in these conditions and none of them is capable of sustaining operations of weeks or months.

The solution proposed is to configure the molten regolith electrolytic reactor into a cold-wall reactor akin to a Hall-Héroult cell used to produce Aluminum industrially. The dimensions of the cell are defined to enable the reactor to heat itself via Joule heating of the melt by the electrolytic current. Such dimensioning relies on the interactions of several variables including the inter-electrode gap, the electrode surface areas and the changes in the melt physical properties to achieve the proper thermal management. In the case of a Hall-Héroult cell, the result is a reactor measuring tens of meters in width, with an inter-electrode gap on the order of $4-5 \mathrm{~cm}$ and an accumulating layer of solid cryolite salt on the reactor walls that protects the structural containment.

Any attempt to determine the parametric values needed to achieve self-heating of a molten regolith electrolytic reactor through experimentations would quickly become prohibitively expensive. We selected a multiphysics simulation software tool, called COMSOL Multiphysics ${ }^{\mathrm{TM}}$ to help solve the problem; it allows users to perform all the steps in the modeling process that include physics and equation definition, mesh generation, equation solving, visualization, and post processing.

\section{Model of a Joule-heated Reactor for Molten Regolith Electrolysis}

\section{A. Physical Model of Reactor}

The primary objective of the modeling effort being to identify the parameters that drive the dimension of a selfheating reactor, a simplified concept was adopted for the reactor (Fig.1). The initial dimensions chosen for the reactor are at the low end of what is expected and were selected based on the computing power available at this time to solve the heat transfer problem within a given volume of material. The model reactor consists in a steel cylindrical shell (ID: $40 \mathrm{~cm}$, height: $10 \mathrm{~cm}$ ) closed at one end containing a bed of regolith (colored pink in Fig. 1.a and violet in Fig. 1.b) and two electrode plates facing each other in a horizontal arrangement. The plates, made of iridium are positioned at the center of the bed and are kept initially at a distance of $2.5 \mathrm{~cm}$ from each other. This 
initial inter-electrode distance is chosen based on experimental data and kept in the same order of magnitude as that of a HallHéroult cell for comparison. An atmosphere of standard air was assumed above the regolith bed.

The upper electrode $(13.5 \mathrm{~cm}$ in diameter) is the anode where oxygen is formed and the lower electrode is the cathode where metals are formed and collected in molten form $(16 \mathrm{~cm}$ in diameter). The anode is immersed in the regolith at half of its 2.5 $\mathrm{cm}$ thickness while the cathode of the same thickness is fully immersed in the regolith. The electrical lead and collector connect the anode and cathode respectively and are made of iridium with a diameter of $5 \mathrm{~cm}$. The boundary conditions imposed in the model include convective and radiative heat transfer between all outer surfaces and the surroundings; thermal radiation between facing surfaces in the cell is also included in the model. The external surface of the bottom of the steel shell is assumed to be thermally and electrically insulated. For each case studies, a constant voltage is set at the top end of the anode lead and the respective ground is set on the bottom end of the cathode collector. Electrical insulation is assumed for all surfaces of the steel shell, meaning that no electrical current passes between the shell surfaces and the regolith and surroundings. Electrical current is generated at the top of one electrode connector, travels through the connector passing through the electrode plates and
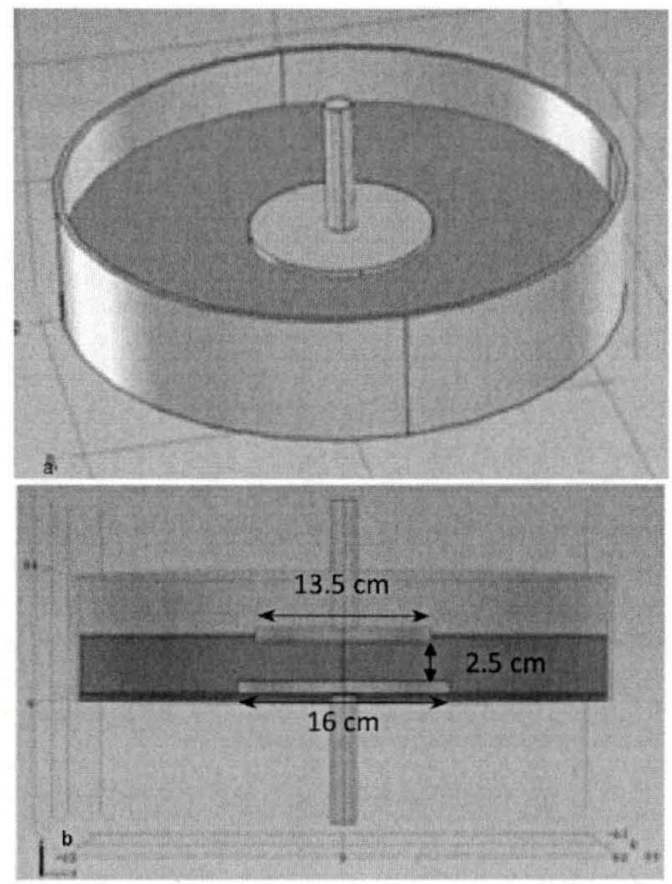

Figure 1. Schematic of Joule-heated reactor used for model. the regolith to continue through the opposite connector.

\section{B. Heat Transfer Model}

As the regolith melting process takes place at temperature higher than $1000{ }^{\circ} \mathrm{C}$, thermal radiation becomes a predominant term in heat transfer adding to conduction and convection and must be included in the model to predict both the temperature profile and the formation of the molten phase accurately. The model includes the following three types of radiative heat transfer; within the regolith, between reactor surfaces and regolith surfaces, and between reactor and regolith surfaces and the ambient. Since the source of thermal energy in the electrowinning reactor is the electrical current passing between the electrodes at a given potential, the resulting Joule heat is expressed to relate electrical current with the heat transfer formulation in three dimensions. Solving for the temperature and the electrical potential, the Joule-heating term is added to the general heat transfer mathematical formulation for solids expressed in Eq. 1 .

$$
\rho C_{p} \frac{\partial T}{\partial t}-\nabla \cdot(k \nabla T)=Q
$$

With the following materials properties:

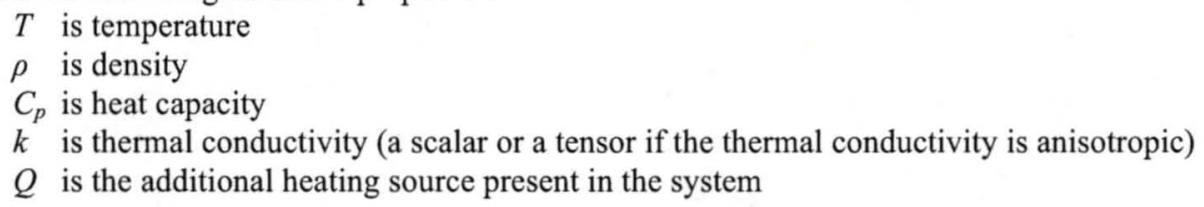

In the model, $Q$ includes two heating sources, Joule heating and thermal radiation in participating media (Eq. 2).

$$
Q=Q_{j}+Q_{r}
$$

Where

$Q_{j}$ is the Joule heating

$Q_{r}$ is the thermal radiation in participating medium 
Joule heating generation $Q_{j}$ is a function of the electrical current applied to the material and the resistivity of the material as shown in Eq. 3.

$$
Q_{j}=\frac{1}{\delta} J^{2}
$$

Where

$J$ is the current density

$\delta$ is the electrical conductivity

The thermal radiation in participating medium $Q_{r}$ uses the radiative transfer equation (Eq. 4) to determine the local thermal radiative intensity, $I$ for each position within the material at all directions. The sum of these local radiative intensities leads to the determination of the total incident radiation $(G)$ within the participating media.

$$
\Omega \cdot \nabla I(\Omega, s)=\kappa I_{b}(T)-\beta I(\Omega, s)+\frac{\sigma_{s}}{4 \pi} \int_{0}^{4 \pi} I(\Omega, s) \Phi\left(\Omega^{\prime}, \Omega\right) \partial \Omega^{\prime}
$$

Where

$I(\Omega, s) \quad$ is the radiative intensity at the position $\mathrm{s}$ in the $\Omega$ direction

$\kappa, \beta, \sigma_{\mathrm{s}} \quad$ are absorption, extinction, and scattering coefficients

$I_{\mathrm{b}} \quad$ is the blackbody radiative intensity

All the thermal radiative intensities $I(\Omega, s)$ for all positions $s$ and $\Omega$ directions are calculated and used to compute the general incident radiation, $G$ as shown in Eq. 5.

$$
G=\sum_{\Omega} \sum_{s} I(\Omega, s)
$$

Finally, the radiative heat source term $Q_{\mathrm{r}}$ is computed as shown in Eq. 6 and its value added in the heat transfer equation (Eq. 2).

$$
Q_{r}=\kappa\left(G-4 \sigma T^{4}\right)
$$

Where

$G$ is the incident radiation within the participating media calculated in Eq. 5

$\kappa$ is the optical absorption coefficient

$\sigma$ is the Boltzmann constant

The model treats surface-to-surface thermal radiation as an energy transfer between boundaries where the medium does not participate in the radiation (radiation in transparent media). The process transfers energy directly between boundaries. The radiation therefore contributes to the boundary conditions rather than to the heat equation itself. In the same way, the model treats surface-to-ambient thermal radiation as an energy transfer between boundaries and the ambient. Another contribution to the heat transfer at boundaries exposed to the ambient is the free convective heat transfer due to the ambient temperature and external fluid.

\section{Properties of Molten Basalts}

The key physical properties required to model the electrolytic self-heating reactor are thermal conductivity, electrical conductivity (resistivity), heat capacity, and density as functions of temperature. The thermal radiation terms require additional physical properties, such as optical absorption and scattering coefficients, refractive index, and thermal emissivity. 
Thermal conductivity and heat capacity of FJS-1 regolith simulant fabricated by the Japanese Aerospace Agency (JAXA) have been reported by others as measured up to $1000{ }^{\circ} \mathrm{C}$ and $800{ }^{\circ} \mathrm{C}$ respectively ${ }^{3}$; values for higher temperatures (glass and molten phases) were extrapolated by us and compared with known experimental values of minerals with similar composition. The thermal conductivity of FJS-1 at low temperature agrees with values measured on the lunar surface ${ }^{4}$ by astronauts of Apollo 17; thermal conductivity of synthetic Apollo 11 sample $^{5}$ measured at high temperature were used along with values found for FJS-1 for temperatures below $1000^{\circ} \mathrm{C}$ to build the temperature-dependent thermal conductivity fitting curve used in the model and shown in Fig. 2.

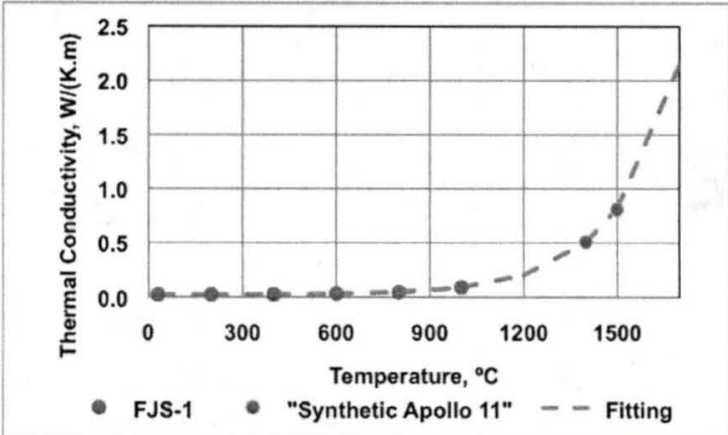

Figure 2. Thermal conductivity of regolith simulants as a function of temperature.



Figure 3. Electrical conductivity of regolith simulants as a function of temperature.

Experimental electrical conductivity of lunar sample 12002,85 measured by Olhoeft et al. ${ }^{6}$ at temperatures lower than $1000{ }^{\circ} \mathrm{C}$ were used in the model along with the thermal conductivity of Tholeiitic basalt measured by Shekhar ${ }^{7}$ within the $700-1700{ }^{\circ} \mathrm{C}$ range. As shown in Fig. 3, the electrical conductivity values of the lunar sample 12002,85 measured at the upper limit of its temperature range overlaps with the values of Tholeiitic basalt measured at the lower limit of its temperature range.

Optical absorption values on molten lunar samples are not readily available. Initially, we selected data provided by Sarkar $^{8}$ who has reported optical absorption coefficients in the $400-760 \mathrm{~nm}$ wavelength region for lunar glassy spherules in an attempt to understand the thermal history of lunar glassy spherules at the moon surface. No other data was found on molten lunar materials over an wider range of wavelengths. We then turned to data produced in glass research for industry. Optical absorption coefficients of molten glass have been measured by several researchers (as function of wavelength) due to its extensive industrial use; molten glass is the material with known optical absorption coefficients closest to molten regolith. Figure 4 shows the absorption coefficient of molten glass experimentally measured at $1,200^{\circ} \mathrm{C}$ and reported by Ninjtten; absorption coefficient of solid glass at $25^{\circ} \mathrm{C}$ is also plotted in Fig. 4 for comparison purpose. As indicated in Fig. 4, the optical absorption coefficient of molten glass at $1,200{ }^{\circ} \mathrm{C}$ averaged 0.25 and $4 \mathrm{~cm}^{-1}$ within the $1,000-2,750$ and $2,750-4,250$ $\mathrm{nm}$ wavelength ranges respectively. As specified in equation 4 , the model assumes that the molten regolith is a gray-type material (optical absorption coefficient is wavelength independent); the absorption value used in the model is $0.25 \mathrm{~cm}^{-1}$ that corresponds to the absorption coefficient of molten glass within the $2,750-4,250 \mathrm{~nm}$ wavelength range. Scattering phenomenon, the radiative energy part that in a particular direction is redistributed over beams in other directions is present the model (see Eq. 4).

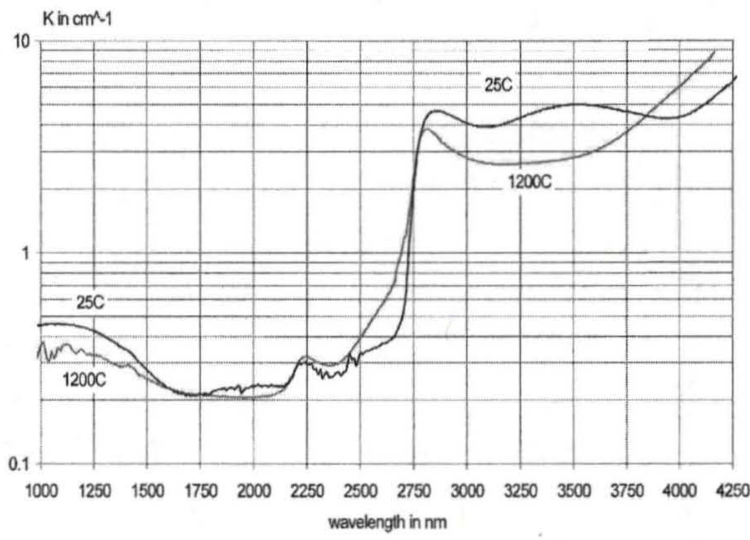

Figure 4. Optical Absorption coefficient of molten glass at $1,200^{\circ} \mathrm{C}$.

During glass production scattering occurs in two stages, the first is when the batch products enter the furnace. As long as the elements in this batch are not completely molten, they represent small spherical particles that redistribute the intensity, much alike water droplets in clouds do with sun light. The other example of scattering in glass production is in the presence of gas bubbles in the glass melt. These bubbles too, redistribute the radiative energy.

5

American Institute of Aeronautics and Astronautics 
The scattering coefficient is currently set to zero in the model as the melt is assumed to not have scattering sources, such as bubbles, voids, solid particles, etc.

\section{Model Validation Case}

The potential of this model to become a predictive tool for reactor design rests on the validation of the results obtained. Since no self-heating reactor has yet been constructed, the experimental results of the irradiation of a regolith bed by a concentrated solar-beam were selected for this purpose. The experiments performed by Gustafson et al. result in the formation of half-spheres of molten regolith during the carbothermal reduction of the oxides under an atmosphere of methane ${ }^{9}$. Figure 5 shows two images of the solar beam generated by a solar concentrator built by Physical Sciences Inc. (PSI) and used by Gustafson to melt the regolith simulant forming a semi-sphere molten puck. Once solidified post-experiment, these half-spheres are collected and measured to obtain physical dimensions and their volume (Fig. 6). The configuration of these experiments is well suited to provide a benchmark to validate the heat transfer model. The profile of the beam is known from in-situ measurements and provides an accurate map of the energy input radiated into the surface of the regolith.

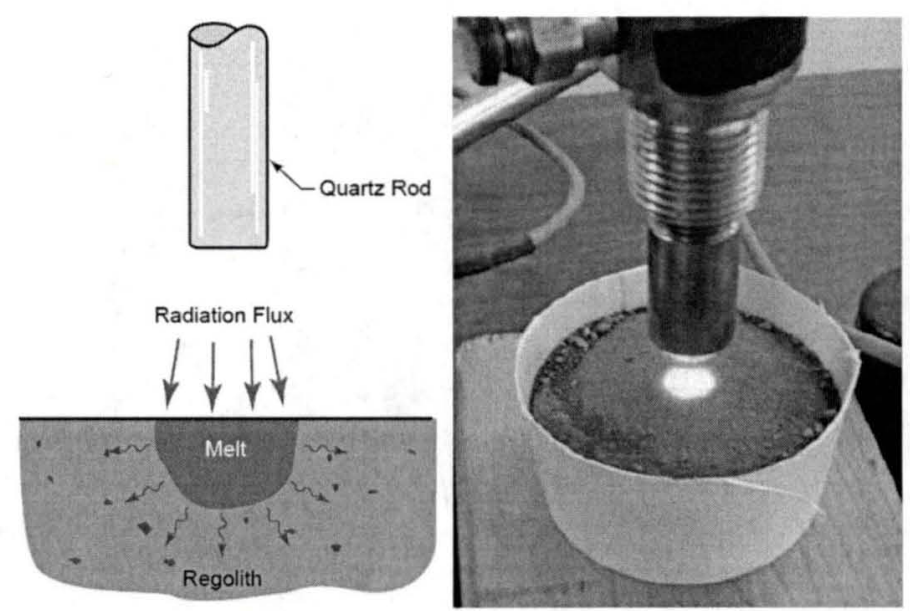

Figure 5. Solar beam irradiation used to melt half-spheres in regolith (Orbitec/Physical Sciences Corp.)

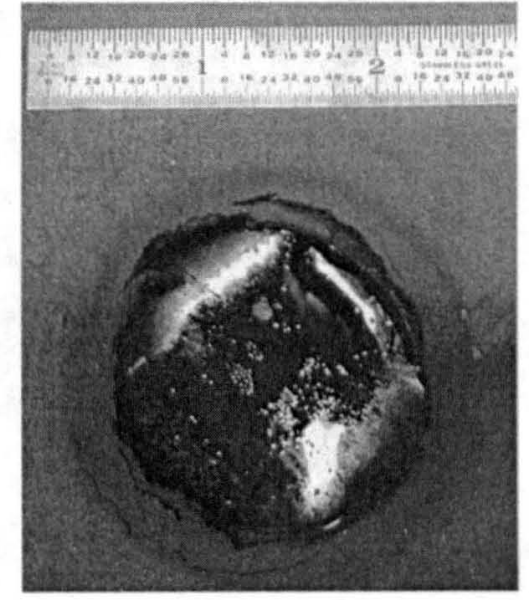

Figure 6. Solidified half-sphere produced by focused solar beam (Orbitec/PSI Corp.)

Using all the temperature-dependent physical properties described above along with the actual solar radiation flux profile yielded at the regolith surface and measured by PSI (the solar concentrator manufacturer), the model was able to predict the dimension of the semi-sphere. Radiative heat transfer in participating media without scattering contribution and thermal radiation to the ambient were taking in account. Figure 7 illustrates the temperature profile when a concentrated solar radiation of $36 \mathrm{~mm}$ in diameter and $1 \mathrm{~kW}$ of output power is used to heat and melt the regolith; red and yellow color represents the molten semi-spherical zone with a diameter of $30 \mathrm{~mm}$, equal to the actual diameter of the melt generated experimentally. The modeled heat transfer conditions also predict an interface between the melt and the granular regolith where a vitrified layer of glass is formed and determines the boundary. This capacity of the model is critical for the prediction of the melt zone in the MRE reactor.

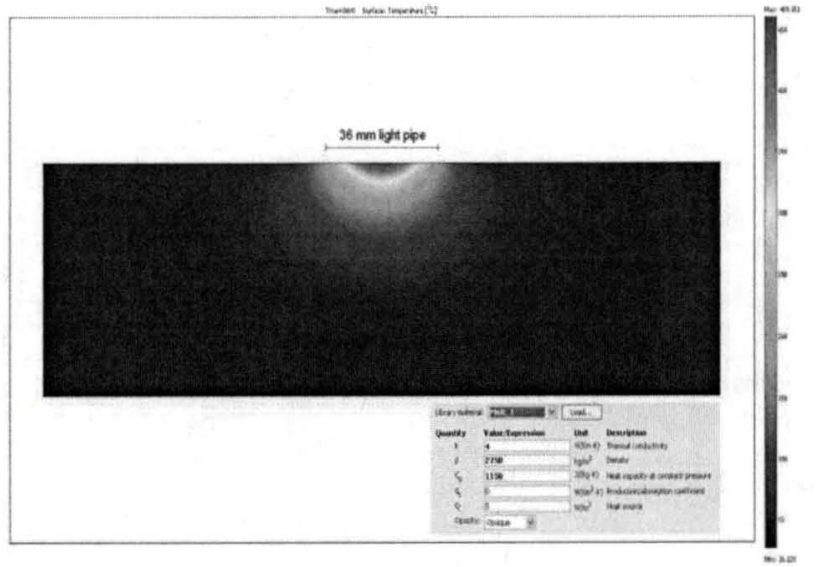

Figure 7. Temperature profile of irradiated JSC-1A melts predicted by the model using Orbitec experimental conditions.

American Institute of Aeronautics and Astronautics 


\section{Results}

The verification of the model performance by the prediction of the experimental irradiated melts gives us the confidence to apply the heat transfer model to the electrowinning cell in molten oxides. The operational values selected to bound the modeled cases come from the experiments successfully conducted at MIT in the last few years.

\section{A. Regolith Heating Between Electrodes ("Cold" Start-up Mode)}

In a validation test of the model, we verified that initiating a "cold start" of a MRE cell by applying only a voltage between the electrodes in an attempt to melt the regolith by Joule heating is not feasible. The thermal energy density and flux needed to melt the regolith cannot be transmitted to the granular material due to its poor electrical and thermal conductivities. We examined cases for different potentials/currents applied to the cell at ambient temperature and run the model to equilibrium without any other heating source but Joule heating. When potentials as high as $50 \mathrm{~V}$ are applied to the top part of the anode connector yielding about $25 \mathrm{~V}$ at the regolith region between the two electrodes, we observed that the calculated Joule heating only generates a small increment in temperature (from 25 to $38{ }^{\circ} \mathrm{C}$ ). For comparison, the thermal conductivity of the regolith at $25^{\circ} \mathrm{C}$ is $0.02 \mathrm{~W} / \mathrm{K} . \mathrm{m}$, a lower value than some of the best performing thermal insulation materials commercially available that have thermal conductivity values between 0.035 and $0.16 \mathrm{~W} / \mathrm{K} . \mathrm{m}$.

\section{B. Molten regolith by Joule heating (internal-heating mode)}

Since the thermal conductivity of the regolith increases substantially at high temperature, going from 0.02 $\mathrm{W} / \mathrm{K} \cdot \mathrm{m}$ at ambient temperature to $3.8 \mathrm{~W} / \mathrm{K} \cdot \mathrm{m}$ at $1400{ }^{\circ} \mathrm{C}$, we focused on evaluating the performance of the cell to maintain a molten electrolyte between electrodes via Joule heating at high temperatures. In this test scenario, the volume of regolith between the electrodes is assumed to be initially in a molten state using an external heating source (preheating) to reach high temperature. The unspecified external heating source was added to the model to start up the heating until the regolith temperature reaches $1700{ }^{\circ} \mathrm{C}$ (molten phase) and is then switched off to initiate electrolysis, which in turn generates Joule heat.

\section{Flat Anode Geometry}

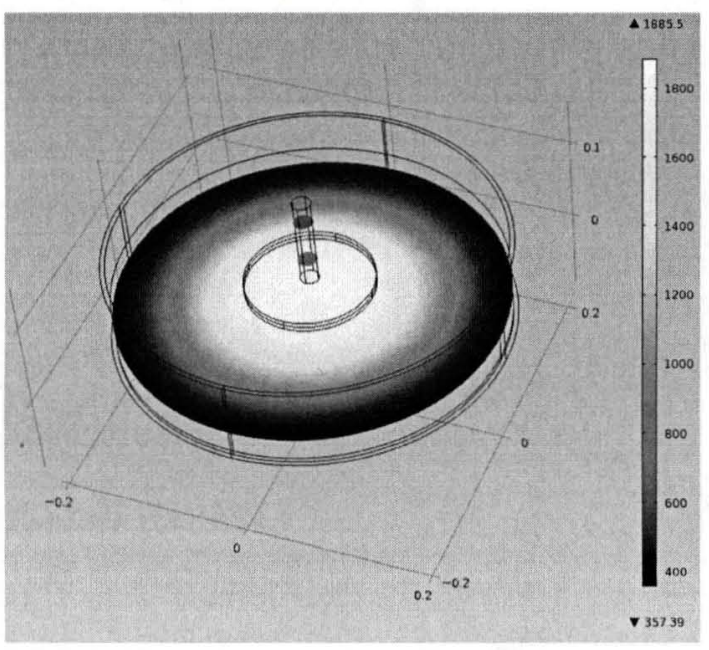

Figure 8. Temperature profile on a single slice of regolith under Joule-heating (with external preheating to $1700^{\circ} \mathrm{C}$ ).

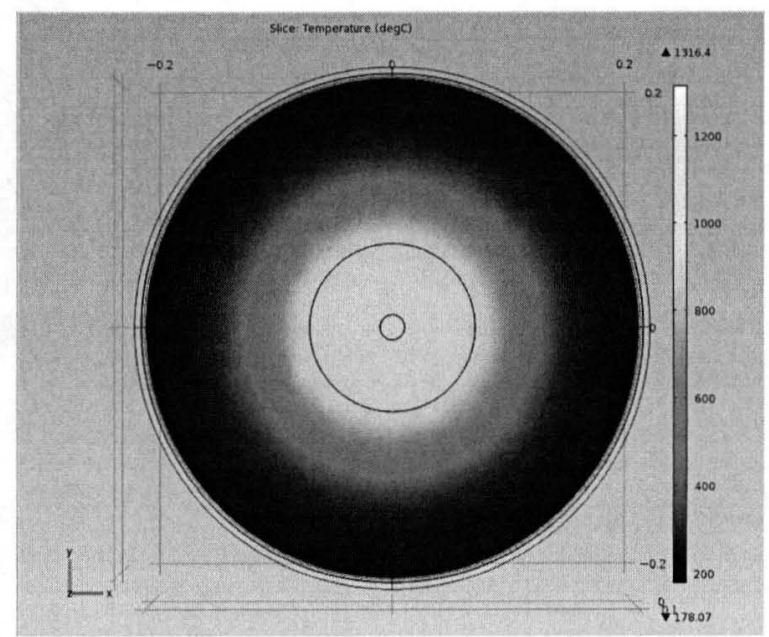

Figure 9. Temperature profile throughout a single slice of regolith under a flat-type anode at $34 \mathrm{~V}$.

Figures 8 and 9 show the temperature profile throughout a single horizontal slice of regolith seeing maximu core temperatures between two flat-disk electrodes. In Fig. 8, a voltage of $50 \mathrm{~V}$ is applied at the top end of the anode lead (resulting in $\sim 25 \mathrm{~V}$ at the regolith section between the two electrodes) and $34 \mathrm{~V}$ is applied similarly in Fig. 9 ( $16 \mathrm{~V}$ at the regolith). It is clear that Joule heating can provide the thermal energy needed to keep the electrolyte in molten phase if the molten pool is formed prior to electrolysis. The temperature at the core of the temperature slice 
in Fig. 9 is $1,316^{\circ} \mathrm{C}$. The corresponding current density calculated by the model is $\sim 0.4 \mathrm{~A} / \mathrm{cm}^{2}$, which is sustainable by the molten oxide mixture based on experiments.

The optical absorption coefficient is a key value to estimate radiative heat transfer within the regolith material at high temperature. Among the physical properties of regolith material investigated in the literature, absorption coefficient is largely left unreported. A coefficient value of $100 \mathrm{~m}^{-1}$ was used in the model based on data reported by Sarkar who measured absorption coefficient of lunar glassy spherules at wavelengths between 400 and $760 \mathrm{~nm}$. Figure 10 shows two images of molten phases to illustrate the effect of the optical absorption coefficient on the temperature profile and the formation of molten phase; the absorption

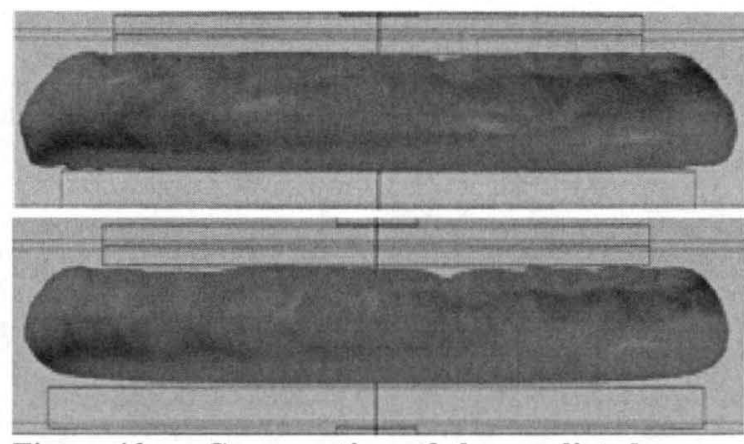

Figure 10. Cross-section of the predicted mass of molten regolith between flat-type anode and cathode during Joule-heating for two optical absorption coefficient values; $5 \mathrm{~m}^{-1}$ (top) and $100 \mathrm{~m}^{-1}$ (bottom). coefficient values of the top and bottom images are 5 and $100 \mathrm{~m}^{-1}$ respectively with identical conditions within the electrolytic cell (i.e., $1,700^{\circ} \mathrm{C}$ initial temperature and $34 \mathrm{~V}$ applied).

The two images clearly show that the model predicts what is expected; the volume of molten material increases with lower absorption coefficient values. Correspondingly, the diameter of the molten phase is extended a bit further.

\section{Waffle Anode Geometry}

The flat-anode geometry is not viable in reality; it does not allow the escape of oxygen at high enough rate and it has a high mass to surface area ratio. At operating temperatures ca. $1600{ }^{\circ} \mathrm{C}$, oxygen occupies very large volumes and tends to be trapped under flat surfaces surrounded by the melt and its presence displaces the melt away from the electrode surface, thus killing the process. We designed a second-generation anode by adopting a waffle geometry that was tested in MRE at a smaller scale. Made of iridium, the modeled anode has a diameter of $14 \mathrm{~cm}$ with holes of $1 \mathrm{~cm}^{2}$ and is made of crossing $2 \mathrm{~mm}$-thick iridium plates connected electrically by five iridium leads (Fig. 11).

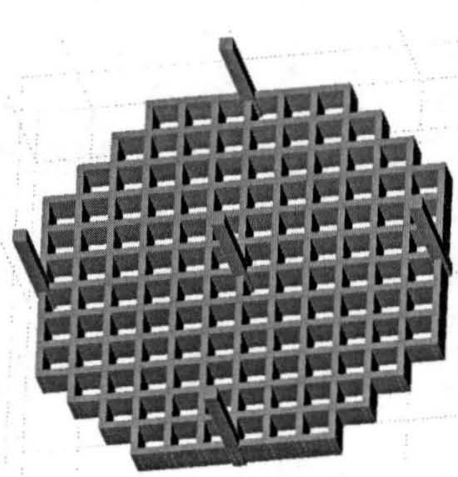

Figure 11. Waffle-anode designed based on experiments to maximize surface area and allow passage of oxygen. Inset: detail of electrical connection of iridium lead and plates.

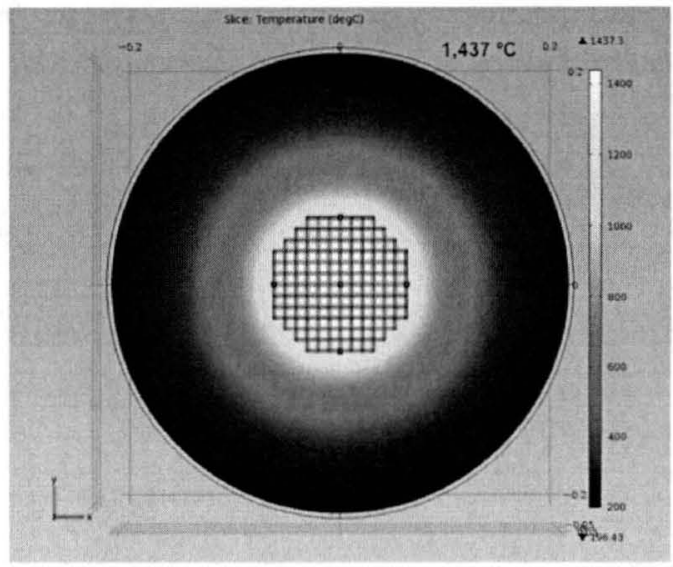

Figure 12. Temperature profile throughout a single slice of regolith under a waffle-type anode at $34 \mathrm{~V}$.

It has a surface area of $520 \mathrm{~cm}^{2}$ compared to $330 \mathrm{~cm}^{2}$ for the flat anode of similar diameter. Our hypothesis also includes that a waffle anode could allow higher current to be passed in the same melt volume therefore getting closer to the predicted current efficiencies. The design also provides flexibility in making changes to decrease the overall resistance of the circuit by improving on contacts or achieving better current distribution. Such considerations have not been tested and modeled yet. 
Figure 12 shows the temperature profile throughout a single slice of regolith between the two electrodes when 34 $\mathrm{V}(\sim 16 \mathrm{~V}$ at the regolith section between the two electrodes) is applied at the top end of the waffle-type anode lead. The geometry of the anodes is the only difference in the operating conditions that lead to the temperature profiles of Fig. 9 (flat-type anode) and Fig. 12 (waffle-type anode). The core temperature of a regolith slice is $121^{\circ} \mathrm{C}$ higher under the waffle-type anode than under the flat-type anode. The current density at the center of the slice shown in Figs. 9 and 12 show values of 0.225 and $0.3 \mathrm{~A} / \mathrm{cm}^{2}$ (at $16 \mathrm{~V}$ applied to the regolith) respectively indicating that the waffle geometry is predicted to yield higher current density between the electrodes leading to higher Joule heating and thus higher melt temperatures. The total power dissipation predicted by the model through the same slice area between electrodes of Figs. 9 and 12 are $1.6 \mathrm{E} 6$ and $2.9 \mathrm{E} 6 \mathrm{~W} / \mathrm{m}^{3}$ respectively corroborating the benefit of using waffle-type anodes over flat-type anodes of similar diameters.

\section{Critical Size of Joule-Heated MRE Reactors for Space Missions}

The electrical energy necessary to operate a Molten Regolith Electrolysis reactor will be a premium commodity during any surface mission to the Moon or Mars. The demonstration of metals and oxygen production by MRE during a dedicated precursor robotic mission can be accomplished with a small reactor comprised of a resistive furnace with refractory ceramics crucibles containing the molten regolith, but such an operation would be designed for a short one time performance. The longevity of an internally Joule-heated MRE reactor and its higher energy efficiency leads us to search for the minimum reactor size for use on a space demonstration mission.

Several current or planned space power systems provide benchmark values for the amount of electrical power that could be made available for future missions that would include this type of reactor. The fuel cells aboard the Space Shuttle delivered $27.5-32.5 \mathrm{~V}$ and an average total power of $16 \mathrm{~kW}$ while plans made under the recent Constellation program establish surface power systems of with an upper limit of $50 \mathrm{~kW}$.

\section{A. Reactor Start-up}

The electrical properties of the regolith indicate that electrical current cannot be passed through it to generate heat. The model confirms this fact when it shows that the modest rise on temperature in the regolith when increasing voltages are imposed to the plate electrodes is actually due to the heating of the plates themselves. This result indicates clearly that a different mode of heat generation must be employed to start the reactor. Such finding is in agreement with current practices in Hall-Héroult reactors that are started by short-circuiting the electrodes with a block of graphite that generates the needed heat to melt the initial electrolyte bed. One could conceive of a similar approach to start a MRE reactor on a remote planetary surface but we face the problem of finding a material that can be consumed in such manner and recycled in-situ.

Direct melting of a regolith mass with a diameter slightly larger than the anode can be accomplished by focused solar beams similar to the previously cited experiments. While this scheme could be considered on the lunar surface but not at Mars, it will require a rather large added mass of solar concentrators to the MRE system. Further work on reducing the system mass could make this option attractive.

The use of arc furnace methods or induction heating could also be developed to input the initial heat into the regolith bed. The start-up problem remains to be solved.

\section{B. Joule-Heating of Molten Regolith at Critical Temperature}

The inclusion of participating media in the heat transfer calculations is validated in the irradiated half-sphere case. The model correctly predicts the formation of the melt within the correct dimensions. The choice of the correct values for the properties of the regolith is also important for the prediction of the formation of the "glass rind" at the interface between the melt and the granular regolith. This zone of solidified vitreous melt is critical for the feasibility of using the regolith as a containment vessel for a MRE reactor; it insulates the molten pool while containing it to protect the steel vessel and it does not contaminate the melt. The model in its current state predicts the formation of a glass rind of 1 to $3 \mathrm{~cm}$ in thickness for Joule-heated melts in the MRE reactor for the cases tested at this time.

We have observed that both experiments leading to the formation of small melts by direct solar beam irradiation and the calculations perfomed by the model prove that melts of many sizes can be contained in a regolith bed. However, the Joule-heating method must accomplish several goals: 1) maintain regolith in its molten state; 2) achieve a critical temperature to keep the metal products as liquids; 3) achieve the best possible energy efficiency as a balance between high current efficiency to maximize production and thermal efficiency for the melt that translates into wasted electrical current (i.e., non-producing current). 
We consider the melting point of iron at $1,535^{\circ} \mathrm{C}$ to be the bottom value for the operational temperature of the reactor to make sure that the metal products at the cathode remain in liquid form. The critical temperature of 1,650 ${ }^{\circ} \mathrm{C}$ is chosen to ensure an acceptable margin based on experimental knowledge. The previous operational examples in Figs. 9 and 12 did not achieve this critical temperature for the entire electrolyte mass between the electrodes. We can vary both the diameter of the electrodes and the distance between them to find a balance between higher overall electrical resistance of the melt, which achieves higher values of Joule-heating and acceptable power efficiency to maintain production of metal and oxygen.

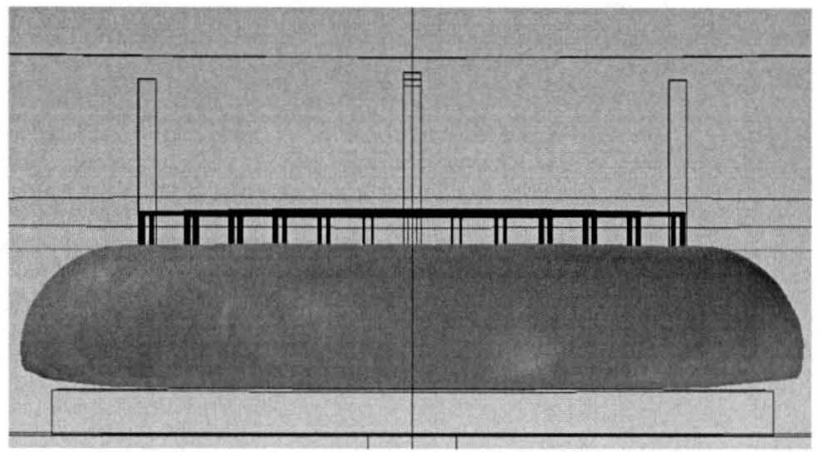

$$
\begin{array}{lr}
\text { Potential (V): } & 34 \\
\text { Current (A): } & 17.6 \\
\text { Power (kW): } & 3.0 \\
\text { Power (kW/kg): } & 0.39 \\
\text { Melt Temp }\left({ }^{\circ} \mathrm{C}\right): & 1,650
\end{array}
$$

Figure 13. Melt temperature profile between waffle-type anode $(11.8 \mathrm{~cm} \varnothing)$ and flat cathode separated by $3 \mathrm{~cm}$.

We identified an example of such balance in the example illustrated in Fig. 13. The diameter of the waffle anode was decreased and the inter-electrode gap was increased generating higher heat flux in the melt without increasing the power required in the system. This combination was found to be advantageous over combinations of smaller inter-electrode gaps and larger anodes.

The current densities produced by the model in this example are within the range of actual experimental values ca. $0.4 \mathrm{~A} / \mathrm{cm}^{2}$, but they are obtained with higher voltages near $16-20 \mathrm{~V}$ in the melt. In this model, the imposed voltage at the plates dictates the magnitude of the electrical current with no constraint on the values of current densities in the model. The voltage values recorded experimentally are below $10 \mathrm{~V}$ and the higher values of the model may be rooted in the values for electrical conductivities selected from Ref. 7 and the absence of modeling of the electrochemical reactions. Such voltage values also mean that a lot of metal oxides present in the regolith with be co-reduced and the resulting metal product will be an alloy of many metals with iron as the main component. Within these constraints, our preliminary findings indicate that a $3 \mathrm{~kW}$ surface power source may be sufficient to operate a Joule-heated MRE reactor on the order of a cubic foot in volume capable of yielding large quantities of oxygen and iron alloys.

\section{Conclusion}

The modeling of all modes of heat transfer within an internally Joule-heated Molten Regolith Electrolysis reactor can be a useful tool to investigate the parameters driving its design. The multiphysics model was validated through the prediction of melt size and a solidified glass rind at the interface of melt and regolith with a similar thickness to those observed in regolith melts formed by solar-beam irradiation. The experimental current densities obtained in externally heated hot-wall reactors have been found as well as the result of the model calculations with some variations. The combination of high surface area geometries for anodes, distributed electrical connections and adjustments of inter-electrode gaps were found to have strong effects on the overall power efficiency performance and thermal performance of Joule-heated MRE reactors for electrolytic reduction of lunar oxides from their own melt. Preliminary findings suggest that the minimum critical size of such reactor may be on the order of a cubic foot in volume with a power requirement of less than $5 \mathrm{~kW}$.

The accuracy of the model is strongly dependent on important variables such as optical properties of the melt, which have yet to be measured for these complex oxide mixtures. The engineering of prototype reactors designed to process regoliths in space at melting temperatures will require the knowledge of these and other fundamental properties of the various mineral resources. 


\section{Acknowledgments}

The authors wish to thank NASA Exploration Technology Development Program and the ISRU Project for funding this effort and NASA Kennedy Space Center.

\section{References}

\footnotetext{
${ }^{1}$ Sibille, L., Sadoway, D. R., Sirk, A., Tripathy, P., Melendez, O., Standish, E., Dominguez, J. A., Stefanescu, D. M., Curreri, P. A., and Poizeau, S., "Recent Advances in Scale-Up Development of Molten Regolith Electrolysis for Oxygen Production in Support of a Lunar Base," 47th AIAA Aerospace Sciences Meeting, AIAA, Washington, DC, AIAA-2009-659, 2009.

${ }^{2}$ Gmitter, A. J., "Electrochemical Production of Oxygen from Molten Oxides: Influence of Inert Anode Material and Electrolyte Composition," Masters Thesis, Dept. of Materials Sciences and Engineering, Massachusetts Institute of Technology, Cambridge, MA, 2008.

${ }^{3}$ Wakabayashi, S., and Matsumoto, K., "Development of Slope Mobility Test Bed using Simulated Lunar Soil," JAXA Report JAXA_RM-05-003, June 30, 2006.

${ }^{4}$ Apollo 17 Preliminary Science Report, NASA SP-330, 1973.

${ }^{5}$ Murase, T., and McBirney, A. R., "Properties of Some Common Rocks and Their Melts at High Temperatures," Geological Society of American Bulletin, Vol. 84, Nov. 1973, pp. 3563-3592.

${ }^{6}$ Olhoeft, G. R., Frisillo, A. L., Strangway, D. W., and Sharpe, H., "Temperature Dependence of Electrical Conductivity and Lunar temperatures," Earth, Moon, and Planets, Vol. 9, 1974, pp. 79-87.

${ }_{7}$ Shekar, C., "Electrical and Elastic Properties of Basalts and Ultramafic Rocks as Function of Saturation, Pressure, and Temperature," Ph.D. dissertation, University of Hawaii, December 1977.

${ }^{8}$ Sarkar, S. K., "Optical Absorption and Chemical Composition of some Lunar Glassy Spherules," J. Material Science Letters, Vol. 7, No. 3, March 1988, pp. 227-229.

${ }^{9}$ Gustafson, R., White, B., Fidler, M., and Muscatello, A., "Demonstrating the Solar Carbothermal Reduction of Lunar Regolith to Produce Oxygen," 48th Aerospace Sciences Meeting, AIAA, Washington, DC, AIAA-2010-1163, 2010.
} 